\title{
Quantification of Dynamic Model Validation Metrics using Uncertainty Propagation from Requirements
}

\author{
Andrew M. Brown, Ph.D. ${ }^{a}$, Jeffrey A. Peck ${ }^{b}$, Eric C. Stewart, Ph.D. ${ }^{c}$ \\ ${ }^{a}$ Aerospace Engineer, NASA/Marshall Space Flight Center, ER41/Propulsion \\ Structural \& Dynamic Analysis, Huntsville, AL 35812 \\ ${ }^{\mathrm{b}}$ Aerospace Engineer, NASA/Marshall Space Flight Center, EV31/Vehicle \\ Dynamics, Loads, and Strength Analysis, Huntsville, AL 35812 \\ ${ }^{\mathrm{c}}$ Aerospace Engineer, NASA/Marshall Space Flight Center, EV31/Vehicle \\ Dynamics, Loads, and Strength Analysis, Huntsville, AL 35812
}

\begin{abstract}
The Space Launch System, NASA's new large launch vehicle for long range space exploration, is presently in the final design and construction phases, with the first launch scheduled for 2019. A dynamic model of the system has been created and is critical for calculation of interface loads and natural frequencies and mode shapes for guidance, navigation, and control (GNC). Because of the program and schedule constraints, a single modal test of the SLS will be performed while bolted down to the Mobile Launch Pad just before the first launch. A Monte Carlo and optimization scheme will be performed to create thousands of possible models based on given dispersions in model properties and to determine which model best fits the natural frequencies and mode shapes from modal test.

However, the question still remains as to whether this model is acceptable for the loads and GNC requirements. An uncertainty propagation and quantification (UP and UQ) technique to develop a quantitative set of validation metrics that is based on the flight requirements has therefore been developed and is discussed in this paper. There has been considerable research on UQ and UP and validation in the literature, but very little on propagating the uncertainties from requirements, so most validation metrics are "rules-of-thumb;" this research seeks to come up with more reason-based metrics. One of the main assumptions used to achieve this task is that the uncertainty in the modeling of the fixed boundary condition is accurate, so therefore that same uncertainty can be used in propagating the fixedtest configuration to the free-free actual configuration. The second main technique applied here is the usage of the limit-state formulation to quantify the final probabilistic parameters and to compare them with the requirements. These techniques are explored with a simple lumped spring-mass system and a simplified SLS model. When completed, it is anticipated that this requirements-based validation metric will provide a quantified confidence and probability of success for the final SLS dynamics model, which will be critical for a successful launch program, and can be applied in the many other industries where an accurate dynamic model is required.
\end{abstract}

Keywords: Structural Dynamics, Uncertainty Quantification, Uncertainty Propagation, Modal Test, Probabilistic Analysis 


\section{NOMENCLATURE}

$\begin{array}{ll}\text { BME } & \text { Best Model Estimate } \\ \text { DOF } & \text { Degree-of-Freedom } \\ \text { FEM } & \text { Finite Element Model } \\ \text { GNC } & \text { Guidance, Navigation, \& Control } \\ \text { IMT } & \text { Integrated Modal Test } \\ \text { ML } & \text { Mobile Launcher } \\ \text { PDF } & \text { Probability Density Function } \\ \text { rv } & \text { random variable } \\ \text { SLS } & \text { Space Launch System } \\ \text { SRB } & \text { Solid Rocket Booster } \\ \text { UQ, UP } & \text { Uncertainty Quantification and Propagation }\end{array}$

\section{INTRODUCTION}

NASA is developing the Space Launch System (SLS) as the first manned extra-orbital vehicle since the Apollo program. Briefly, the system consists of a Core Stage consisting of a liquid hydrogen and oxygen tank and four RS25 liquid rocket engines; a second stage powered by one RL-10 engines; the manned Orion capsule and command module upper stage; and two advance Solid Rocket Boosters (SRB) attached to the sides of the core stage (see figure 1). As with any launch vehicle, the structural dynamics finite element model (FEM) is vitally important for a number of reasons. First, the dynamic model is critical for the successful design of a safe guidance, navigation, and control (GNC) system, since in-flight vectoring of the propulsion system must be consistent with system mode shapes. Second, the dynamic model is the central basis for the determination of dynamic loads as they propagate from numerous sources at all phases of the mission, which are used for strength analysis throughout the vehicle.

As the above factors are well-recognized, modal testing of launch vehicle systems has always been a requirement for space flight. However, determining the details of this modal testing is problematic; there are many different configurations and system components to consider, and it is difficult to determine which configuration and which combination of components should be tested. In recent years, for instance, the launch dynamics community has started moving away from traditional "full-up" modal testing of the entire system in favor of testing individual substructures (e.g., the SRB's, the Core Stage, etc.) and then analytically tying the test-verified components together. As far as testing the proper configuration, it is very difficult to devise a true "flight" configuration, as this would involve devising accurate free-free boundary conditions and would require loading of the propellants into the fuel tanks, which would be dangerous and extremely costly.

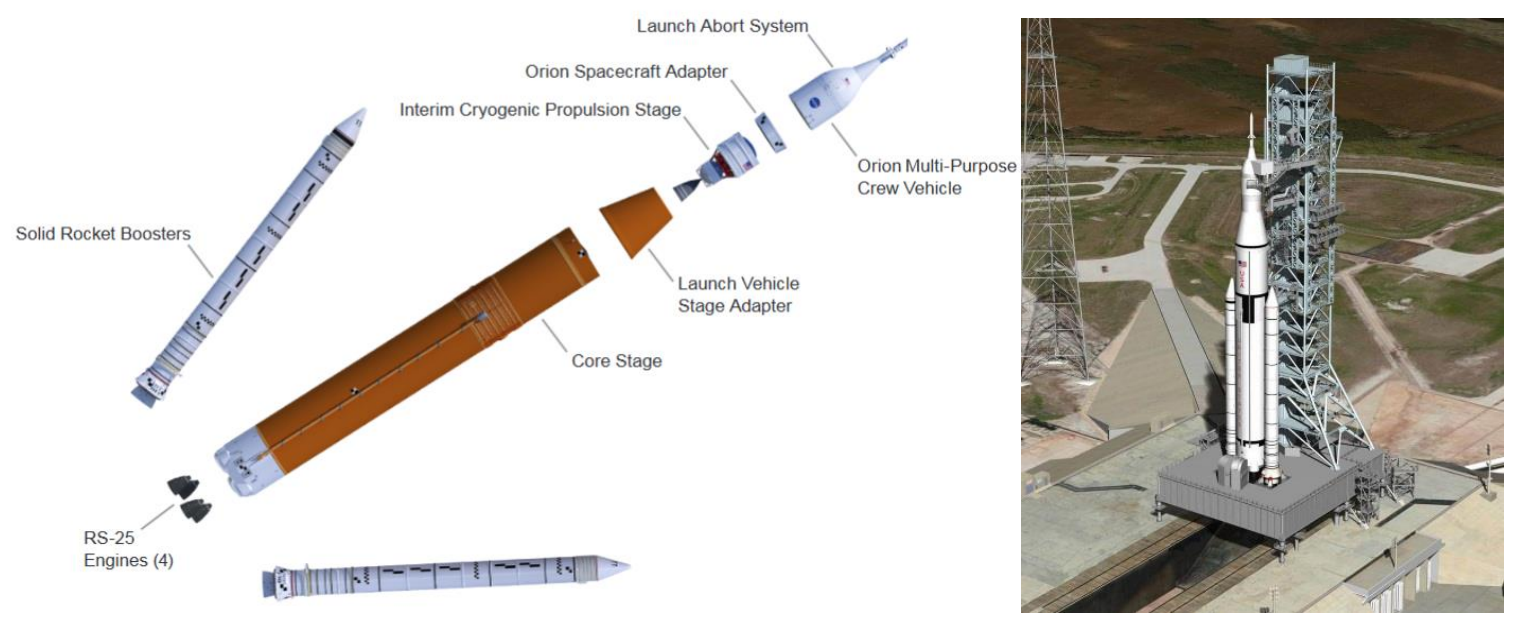

Fig. 1 Space Launch System Block I Configuration and mounted on Mobile Launcher 
The SLS program has made several decisions regarding the above issues. First, the primary modal test of the system (called the Integrated Modal Test, or IMT) will be with the empty SLS mounted on the Mobile Launcher (ML), which itself is a complex structure. The validation of the SLS flight model will be achieved using a probabilistic optimization technique defined as the "Best Model Estimate (BME)" method developed by Stewart [1]. Since the IMT is scheduled for only a couple of months before the first SLS launch, the BME method uses a Monte Carlo analysis of a large pre-determined set of potential SLS models using probabilistic dispersions of primitive geometric variables in the finite element model. Once the IMT is performed, each Monte Carlo sample is compared with the natural frequency and mode shape results, and a Pareto Front is created that identifies the "best model."

Although a best model would be created, though, no criteria had been established for whether that model was actually accurate enough to be acceptable for the applications to GNC and loads. Although typical model correlation criteria such as "less than 10\% variation in natural frequency" and "greater than 0.9 on the Modal Assurance Criteria diagonal" could be applied, these are not tied to the dynamic model requirements.

An effort was therefore initiated to develop a technique that uses the requirements for the dynamic model to generate the metrics for its acceptability. For example, the GNC community has a guideline that says the post-test modeled first bending mode flight frequency must be no less than $-3 \%$ of the pre-test model first bending mode. The first bending mode can increase in frequency from the pre-test to post-test models with little impact on the GNC community, so no guidelines exist for that case. Although there are other important applications for the dynamic model, it is believed that the GNC requirement is the most stringent and provides a clear metric to be met prior to flight. A technique has therefore been developed to address the need for model acceptability metrics and is described in this document; as this is a work-in-progress, the technique has only been implemented on simplified systems and so this paper should be considered an "extended abstract" rather than as a paper with fully-vetted results. Nevertheless, the technique is unique as a "requirements-based metric" so publication at this time should be valuable to the dynamics community.

\section{LITERATURE SURVEY}

There has been substantial work in the literature on uncertainty quantification and propagation (UQ, UP), but little on the reverse propagation that is needed for establishment of the desired metric. Hasselman has published a great deal in this area, including papers on structural dynamics model accuracy[2] and on UQ, focusing on quantifying and propagating structural dynamic model uncertainty using modal test [3], [4]. His UQ papers describe a rigorous methodology using the system frequency response functions as well as mass and stiffness matrices, and the use of a proprietary software code for implementation. Chen in 2004 laid out a step-by-step process for model validation using uncertainty propagation that does have elements considered useful for this research [5]. A metamodel, or Response Surface Model, is used as a surrogate for the original numerical model to enable large sample Monte Carlo simulation, and an assessment of model validity then is obtained by generating a probability density function (PDF) comparing the response and experiment, and identifying the "critical limit of confidence level where the physical experiment falls exactly at the boundary of the performance range obtained from the computer model." This process is very similar to that eventually chosen by the authors for this work. Other valuable work in this field include a number of publications by Kammer that focus on quantifying the uncertainty propagation in substructures when they are coupled together into a system model [6].

\section{METHODOLOGY}

The methodology here somewhat follows the procedure laid down by Chen by creating a limit state of the ratio of the modal test and model primary natural frequency. Our interpretation of the GNC requirement is that the true frequency can be no less than a value that is $3 \%$ less than the pre-test prediction, which comes from the FEM. The performance limit-state ratio is applicable here, therefore, because we assume that the true value of the natural frequency lies within a statistical range defined by a mean equal to the test value obtained by the modal test and a distribution that is a function of sensor placement and other purely test-based errors. 
The other critical concept is an assumption that the statistical distribution of the relationships between the natural frequencies of the modelled ground-modal test model and flight model are accurate, so therefore that same uncertainty can be used in propagating the uncertainties in the actual ground modal test to what we call here a "pseudo-test" flight configuration. This assumption requires that there is a clear shape equivalence between a ground-modal test mode and the critical flight mode upon which the requirement is levied. If this is the case, we assume that the distribution of the ratio between the modeled flight configuration critical frequency and modeled fixed configuration equivalent modal frequency can also be used to relate the tested ground fixed configuration to the "pseudo-modal-tested" flight configuration, .i.e.

$$
\left\{f_{f t}\right\}=\left\{f_{g t}\right\} *\left\{\frac{f_{f m}}{f_{g m}}\right\}
$$

where $\left\{f_{g m}\right\}=$ vector of frequencies of shape equivalent mode from FEM of ground-modal-test, created using Monte Carlo with assumed statistical distributions of parameters; $\left\{f_{f m}\right\}=$ vector of frequencies of critical mode from flight FEM, created using Monte Carlo with assumed statistical distributions of structural parameters; $\left\{f_{g t}\right\}=$ vector of frequencies of shape equivalent mode from ground-modal-test, created using Monte Carlo with assumed statistical distributions of measurements; and $\left\{f_{f t}\right\}=$ vector of frequencies of critical mode from flight configuration extrapolated from modal-test vector.

Using these values, we then formulate the performance limit state as the ratio of the flight "test" frequency (which is really only a "pseudo-test", and which we have defined to be the distribution about the true value) to the flight model frequency, and subtract 1.0 to calibrate it about zero

$$
g=\frac{f_{f t}}{f_{f m}}-1
$$

The PDF of $g$ can be generated directly from the random vectors, and can be integrated from -.03 to $+\infty$ to obtain the probability of "success", i.e., the probability that the model frequency is within the acceptable range of the critical true frequency. If this probability is higher than the value determined by the program authority, the model is "acceptable." It should be noted that although this new metric is based on the physics of the problem, engineering judgment is still necessary to assess what level of success is adequate.

\section{EXAMPLES}

Two examples were created to flesh out the process and to more clearly identify the critical concepts and assumptions used. The first example is a multi degree of freedom (DOF) lumped spring mass system developed and analyzed in Mathematica ${ }^{\circledR}$, as shown in figure 2. The "flight" configuration, which has free-free boundary conditions, is represented by substructure A, which includes the spring $k 1$ to the boundary DOF, while the "groundmodal-test" configuration is simulated with an additional substructure B which is grounded. It was initially thought that the ground test configuration could be represented by simple springs to ground, but it was then realized that since the ground-test fixture was the entire Mobile Launcher (ML, see figure 1), which possesses its own complex structural dynamics, representation by a mere stiffness (or even a number of springs) could not be used. We let all the springs in the system be random variables (rv's) representing modeling, material, and other uncertainties, and assign distribution types, means and standard deviations to each rv. A 10,000 sample Monte Carlo analysis is then performed to obtain random vectors of the model fixed first frequency $\mathrm{flgm}$ and the model free-free second frequency $f 2 f m$ (first flexible mode), using the same sample sets for the shared rv's in substructure A.

At this point a modal test is assumed to be performed on the entire "ground-test-configuration" and the fundamental frequency is obtained. As described previously, due to sensor placement, measurement and other testing errors, it is assumed that this value would be within the range of the true value of this frequency established by the distributions 


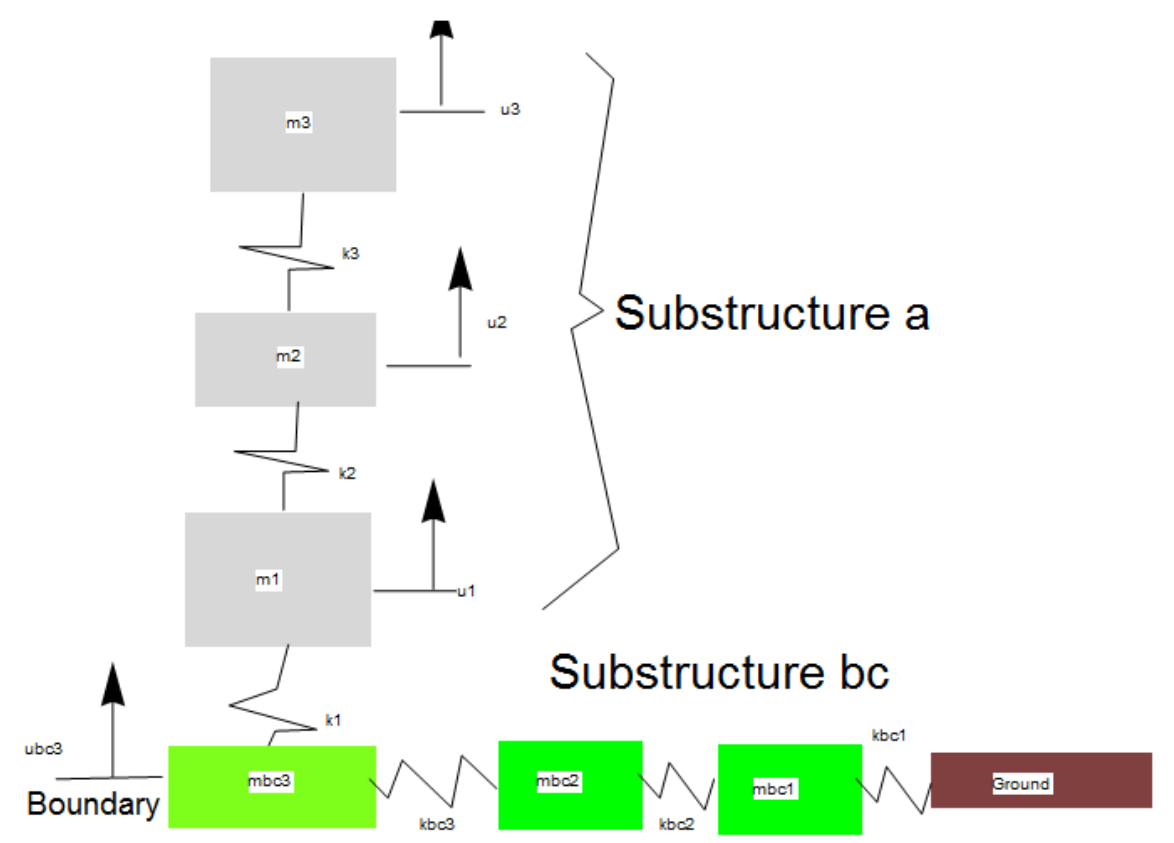

Fig. 2 Discrete MDOF System Representing SLS Vehicle and Mobile Launcher

on these errors. For this MDOF system, we assume these errors follow a normal distribution about the mean, which will be the result from the modal test, and possess a $2 \%$ coefficient of variation. We now use the previous vector of ratios of the "flight" model to the "ground-modal-test" model to extrapolate the "tested" flight frequencies from the tested ground frequencies, i.e., a "pseudo flight-test configuration".

We now create the limit state as the ratio of the flight "test" frequency (which is really only a "pseudo-test") to the flight model frequency, and subtract 1.0 to calibrate it about zero. For the values assumed in this example, the PDF of this limit state is shown in figure 3. The probability of success is defined to be the integral under the PDF from -.03 to + infinity, and is $91.3 \%$ for this set of parameters.

The second example is intended to be a simple but reasonable facsimile of the actual flight and ground-test configuration of the SLS (see figure 4). Approximate lengths and masses were used for the entire system, and the parameters were tweaked to approximately obtain a match with the primary frequency of interest in the actual SLS, which is the third flexible mode at $1.22 \mathrm{hz}$ (here we get $1.38 \mathrm{hz}$ ). This example allows experimentation with the various deterministic and probabilistic software packages necessary for the analysis of the true vehicle. In addition, an additional non-structural mass was added to the "flight" configuration-only to represent the propellant mass. A distribution is assigned to this parameter, but it has to be assumed that this distribution is correct as testing in the flight configuration isn't possible.

The same process as used for the MDOF lumped system was applied to this simplified SLS system model. For this model, the Young's moduli of the SLS core stage, the SRB, the hold-down posts on the ML pad, the ML pad plates, and the upper ML and lower ML main beams, and the propellant mass were established to be independent rv's. The probabilistic wrapper code LS-OPT is used to define the distributions of the random variables and to run NXNastran jobs varying each of the parameters in a Monte Carlo analysis. The analysis on the flight configuration is performed first, and the resulting vector of the primary bending mode natural frequencies saved. The Monte Carlo rv sample set is also saved and the random variables shared with the ground test configuration (all of the SLS substructure except for the propellant) were reused along with the ML substructure rv's in a Monte Carlo analysis of the ground-modal test configuration to obtain the appropriate bending mode closest to the flight mode of interest. A chart output from LS-OPT showing the correlation of the response natural frequency to each of the input random variables is shown in Figure 5. 

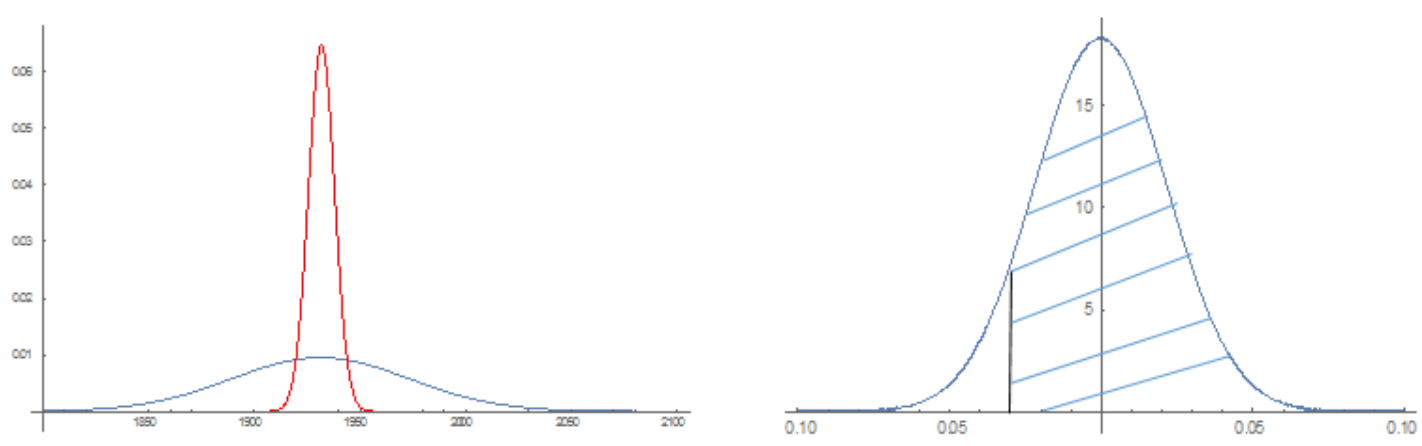

Fig. 3 Example 1 a) PDF's of model frequency (red) and test frequency (blue); b) PDF of Limit State g with success defined by hatched area.
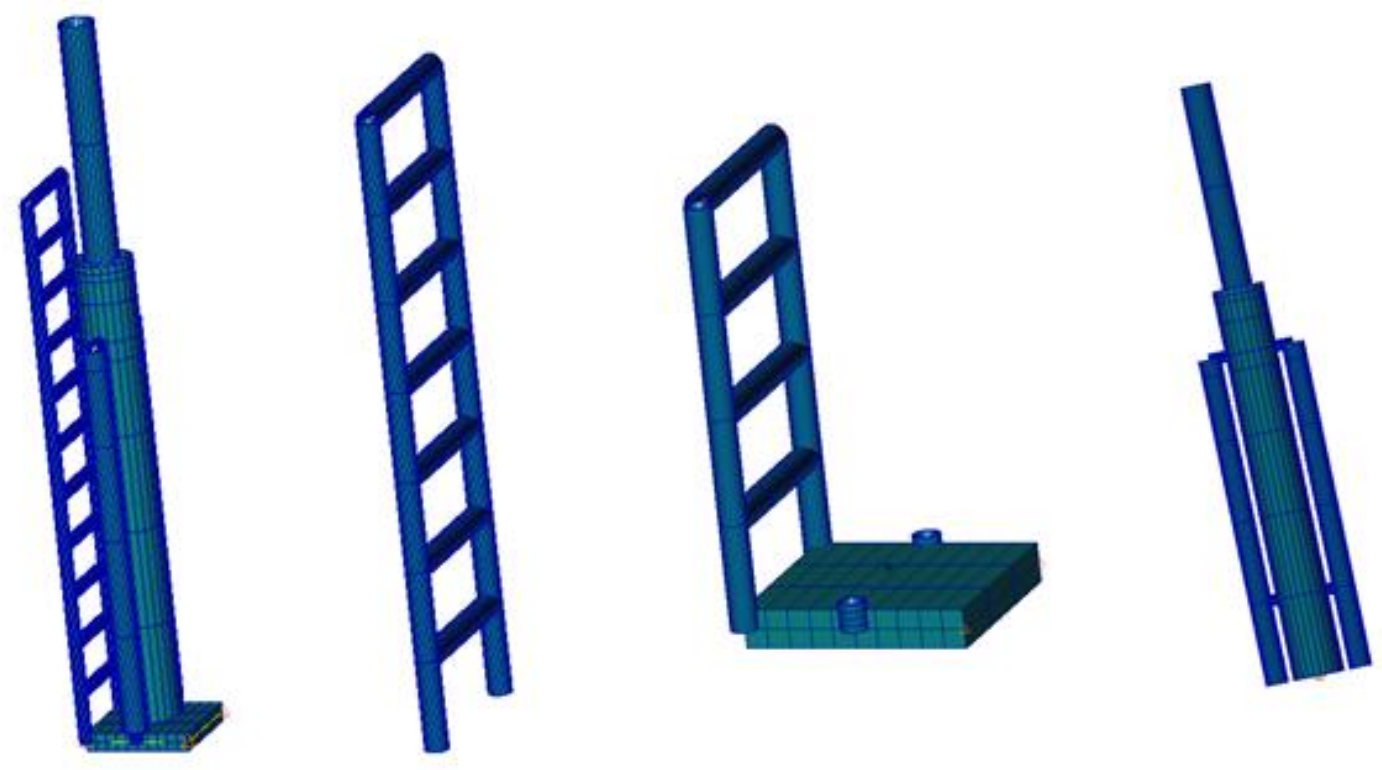

Fig. 4 a) Entire simplified SLS model on Mobile Launcher; b) Upper ML substructure; c) Lower ML substructure; d) SLS substructure (and flight configuration when propellent mass added)

The ratio for each sample from the two MC analyses is then calculated and imported into Mathematica, where the limit state PDF generated and a probability of success is calculated, shown in Figure 6 . In this case, the probability of "success" is only $61 \%$. Figures 5 and 6 introduce several questions which will need to be examined. First, the correlation is almost non-existent between most of the random variables and the response variable, and only weak for one of them; this result indicates that the particular mode of interest is more a function of some other parameter that has not be accounted for. Secondly, looking at a variety of choices for the statistics of the random parameters in these examples, which at this point are completely arbitrary, various success rates point to the fact that the requirements will favor either an overestimation of the natural frequency rather than a more accurate estimation if there is substantial aleatory variability in the test or model, or a small variation in the model variability, which is expected. This is indicated by examining the individual PDF's of the "pseudo-test" versus "model" for the examples (figure $3 a$ and $6 a$ ). 


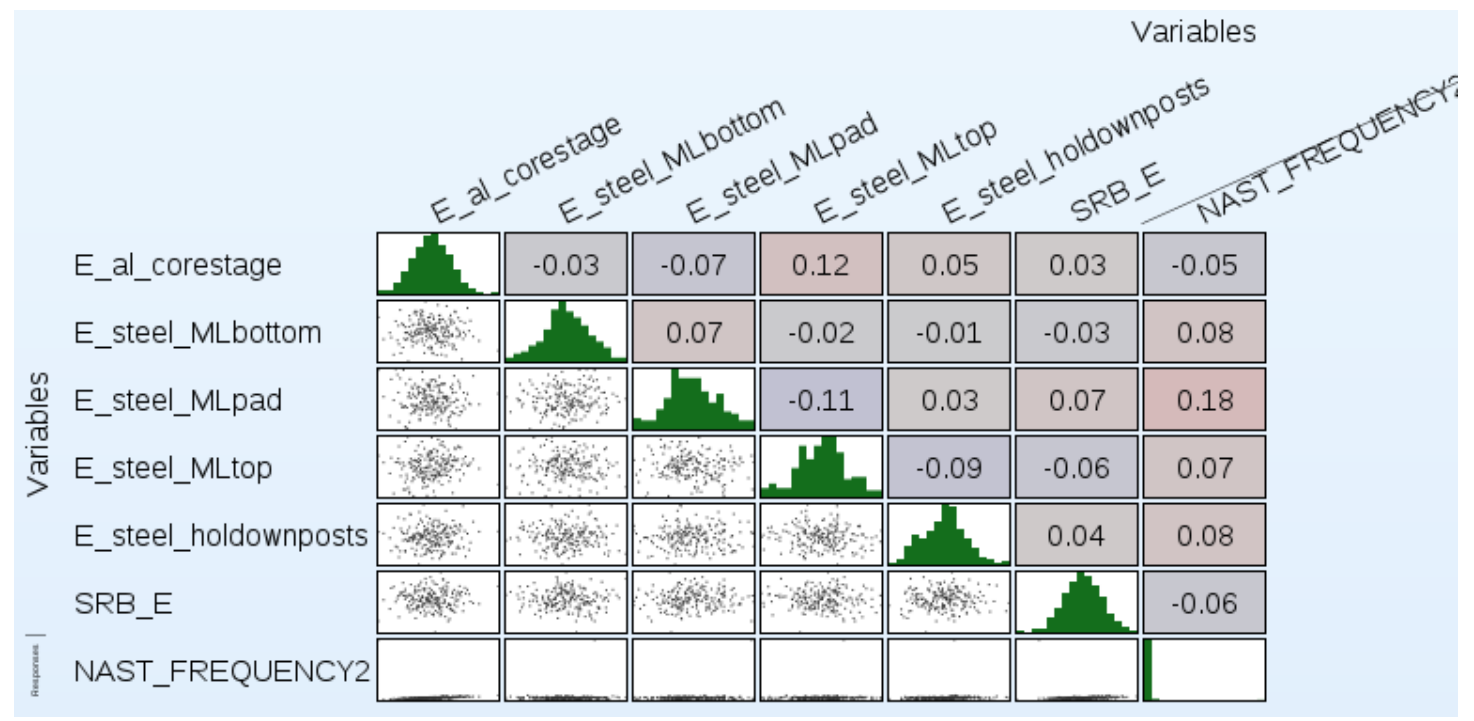

Fig. 5 Correlation Matrix relating Random Variables with Response Variable, and showing Distributions

\section{FORWARD WORK AND CONCLUSION}

A major point of consideration identified is how to implement the random parameters. We presently believe that "primitive" random variables that attempt to approximate actual material and stiffness variations in the structure will be implemented for the vehicle, but that more gross variations on the entire mass and stiffness matrices of the ML will be applied to avoid unnecessary complication; there is no need to perform an excessive amount of analysis on the ML as the only quantity of interest is how it affects the fundamental mode at the boundary with the SLS at the hold-down posts. Another key avenue for investigation, as identified in the simplified SLS model case, is thoroughly understanding the sensitivity of the response variable to each of the random variables. Special attention must be paid to accurately identify the statistical parameters of those input rv's with the highest sensitivity. Finally, an examination is also being undertaken to use techniques developed by Allen [7] to replace the modes of the entire system with purely fixed modes of the vehicle, which would simplify the uncertainty model significantly.

After this decision is made, an attempt will be made to apply the process using the model and test results for the Ares I-X, a 2012 NASA test launch vehicle, to further identify adequacy of the technique. If this is successful, the technique will be applied to SLS as planned with a final deadline in mid-2018. Successful application of this technique would also be an important contribution to the development of metrics for dynamic models, and would mark one of the first attempts at using system requirements to develop these metrics.

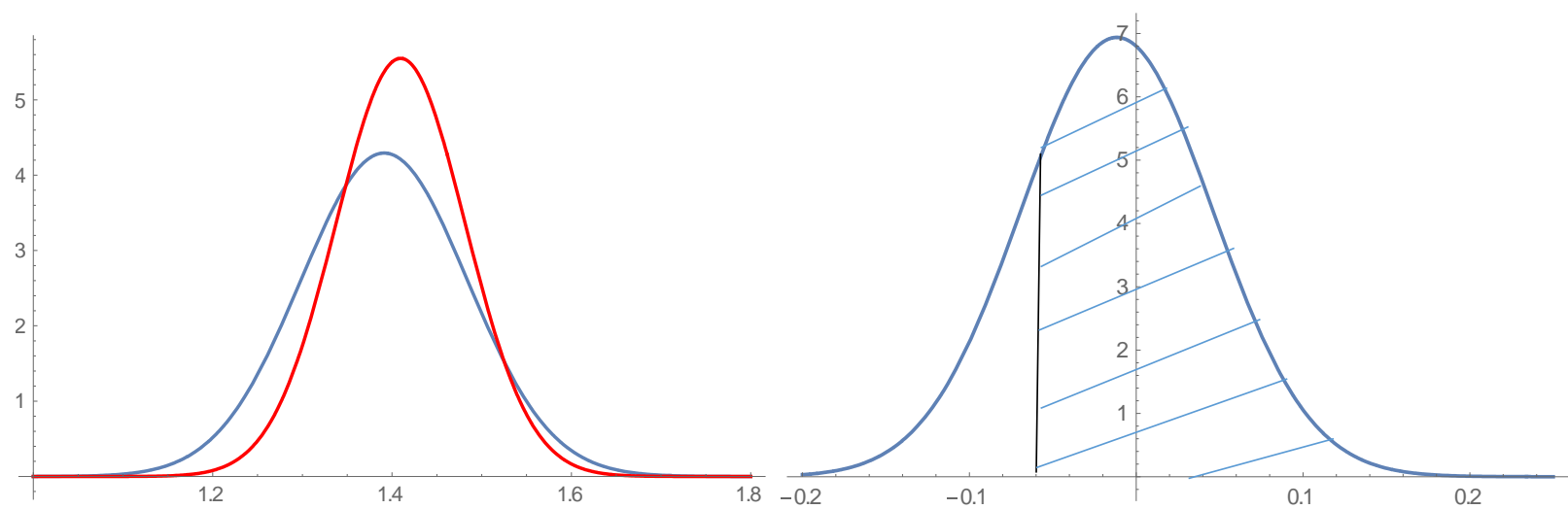

Fig. 6 Example 2 a) PDF's of model frequency (red) and test frequency (blue); b) PDF of Limit State g with success defined by hatched area. 


\section{REFERENCES}

[1] Stewart, E., Hathcock, M., "Using Dispersed Modes During Model Correlation”, AIAA SciTech Forum, 2017

[2] Hasselman, T., Coppolino, R., Zimmerman, D., "Criteria for Modeling Accuracy: A State-of-the-Practice Survey," Proceedings of the 18th IMAC Conference, San Antonio, TX, February 2000.

[3] Hasselman, T., "Effect of Modal Test Statistics on Modeling Uncertainty and Model Updating", AIAA

Structures, Structural Dynamics \& Materials Conference, 2000, Paper 2000-1443

[4] Hasselman, Timothy, "Quantification of Uncertainty in Structural Dynamic Models," ASCE Journal of Aerospace Engineering, October 2001.

[5] Chen, W. ,"Model Validation via Uncertainty Propagation and Data Transforms", 2004

[6] Kammer, D. C., and Krattiger, D., "Propagation of Spacecraft Free-Interface Substructure Uncertainty into System Test-Analysis Correlation," Journal of Vibration and Acoustics, Vol. 134, No. 2, Oct. 2012, 051014

[7] Allen, M. S., Gindlin, A., Mayes, R., "Experimental modal substructuring to estimate fixed-base modes from tests on a flexible fixture", Journal of Sound and Vibration, 330 (2011) 4413-4428 